\title{
Vortex phase boundaries from ferromagnetic resonance measurements in a patterned disc array
}

\author{
C. C. Tsai, ${ }^{1}$ J. Choi, ${ }^{2}$ Sunglae Cho, ${ }^{2}$ S. J. Lee, ${ }^{3}$ B. K. Sarma, ${ }^{4}$ C. Thompson,,${ }^{5}$ O. Chernyashevskyy, ${ }^{5}$ I. Nevirkovets, ${ }^{5}$ \\ V. Metlushko, ${ }^{6}$ K. Rivkin, ${ }^{7}$ and J. B. Ketterson ${ }^{5,8}$ \\ ${ }^{1}$ Department of Engineering \& Management of Advanced Technology, Chang Jung Christian University, Tainan 71101, Taiwan \\ ${ }^{2}$ Department of Physics, University of Ulsan, Ulsan 680-749, South Korea \\ ${ }^{3}$ Department of Physics, Hanyang University, Seoul 133-791, South Korea \\ ${ }^{4}$ Department of Physics, University of Wisconsin, Milwaukee, Wisconsin 53201, USA \\ ${ }^{5}$ Department of Physics, Northwestern University, Evanston, Illinois 60208, USA \\ ${ }^{6}$ Department of Electrical and Computer Engineering, University of Illinois, Chicago, Illinois 60607, USA \\ ${ }^{7}$ Department of Physics, Texas A\&M University, College Station, Texas 77843, USA \\ ${ }^{8}$ Department of Electrical and Computer Engineering, Northwestern University, Evanston, Illinois 60208, USA
}

(Received 23 December 2008; revised manuscript received 6 June 2009; published 22 July 2009)

\begin{abstract}
Using a recently developed broadband microwave measurement technique, we have studied the hysteretic appearance and disappearance with in-plane magnetic field of the uniform ferromagnetic resonance (FMR) mode of a patterned permalloy disk array. The observed features are consistent with our micromagnetic simulations (performed on an infinite array of such disks), which predict that on decreasing the magnetic field from a positively magnetized state at positive fields the array will: (i) pass continuously into a double-vortex state; (ii) followed by a discontinuous transition to a single-vortex state; and finally (iii) discontinuously into a negatively magnetized state at some negative field. The hysteretic counterpart occurs on reversing the field sweep and returning to positive fields. The FMR data are consistent with the hysteretic dc magnetization measurements performed earlier on samples patterned in an identical manner.
\end{abstract}

DOI: 10.1103/PhysRevB.80.014423

PACS number(s): 76.50.+g, 75.70.-i, 75.60.Jk, 41.20.Gz

There has recently been much interest in measuring the ferromagnetic resonance (FMR) spectrum of patterned nanostructured magnetic arrays. As an early example, measurements on square permalloy (Py) disk arrays showed the presence of multiple absorption lines, the structure of which changed with the in-plane field angle, clear evidence of a disk-disk interaction. ${ }^{1,2}$ Measurements have also been carried out on a wide variety of other structures such as rings, ${ }^{3}$ holes, ${ }^{4}$ ellipsoidal holes, ${ }^{5}$ etc. Evolving in parallel have been micromagnetic strategies to calculate the FMR spectrum of nanostructures. Two approaches have proved popular both of which are based on representing nanostructures as discrete dipoles positioned on a lattice each one of which precesses under the influence of static and dynamic, homogeneous or inhomogeneous, external applied fields as well as internal fields arising from exchange, magnetic anisotropy, and the field produced by the remaining dipoles, all evolving according to the Landau-Lifshitz (LL) equation. In the first of these approaches one carries out a brute-force time integration of the coupled LL equations subject to some initial conditions. The widely used NIST OOMMF code is freely available for this task; ${ }^{6}$ alternatively there is the RKMAG code. ${ }^{7}$ The first approach is necessarily slow running and does not yield mode frequencies directly, which must be obtained by analyzing the response; ${ }^{8}$ however it has the virtue of being able to handle the full nonlinear behavior. The second approach linearizes the equations of motion thereby yielding an eigenvalue problem from which all the mode frequencies emerge at once; ${ }^{9}$ codes utilizing this approach are also freely available. ${ }^{7}$ Using the eigenvectors of the associated matrix one can directly calculate the absorption spectrum for an arbitrary harmonic driving field. ${ }^{10}$ The approach was recently generalized to treat the static and dynamic properties of periodic nanostructures. ${ }^{11}$
In disk and ring nanostructures the lowest-energy state is a vortex in which the local magnetization vectors lie on concentric circles in the plane; the vortex core that is present for the case of a disk points out of the plane. The existence of this state has been demonstrated by detecting the absorption from a gyroscopic mode associated with the vortex core, ${ }^{12}$ the frequency of which is in agreement with OOMMF simulations. ${ }^{13}$

The vortex structure can be regarded as a new magnetic phase of a disk array and the present work is concerned with the appearance and disappearance of this phase with external magnetic field. A transition from a uniformly magnetized state to a vortex state can be inferred from the behavior of the measured magnetization as the magnetic field is swept through zero, starting from some sufficiently high value. Figure 1 shows such a measurement on a square array of circular permalloy $\mathrm{Ni}_{0.8} \mathrm{Fe}_{0.2}$ disks as reported by Hoffmann et $a l . ;^{14}$ here the disk diameter and thickness are 600 and 25 $\mathrm{nm}$, respectively, and the interdisk spacing is $1 \mu \mathrm{m}$. The arrays were fabricated on $\mathrm{Si}(100)$ substrates via standard $e$-beam lithography, from an $e$-beam-deposited Py film, using a lift-off process. Unlike a conventional $M-H$ hysteresis curve, the observed behavior is highly asymmetric. On reducing the field from +1000 Oe (see Fig. 1 where the arrows denote the trajectory in the $M-H$ plane) we see that starting at a field of approximately 100 Oe (a nucleation field denoted as $H_{n}$ in the figure) the magnetization first falls rapidly; however near zero field the curve takes on a much smaller slope, ultimately saturating at a field of approximately $-450 \mathrm{Oe}$ (an annihilation field denoted as $H_{a}$ ). Note the curves in Fig. 1 do not cross but rather "kiss" each other near zero field. On increasing from -1000 Oe a similar shift from a larger initial slope to a smaller one on passing through zero is observed. From these data we infer that between about 


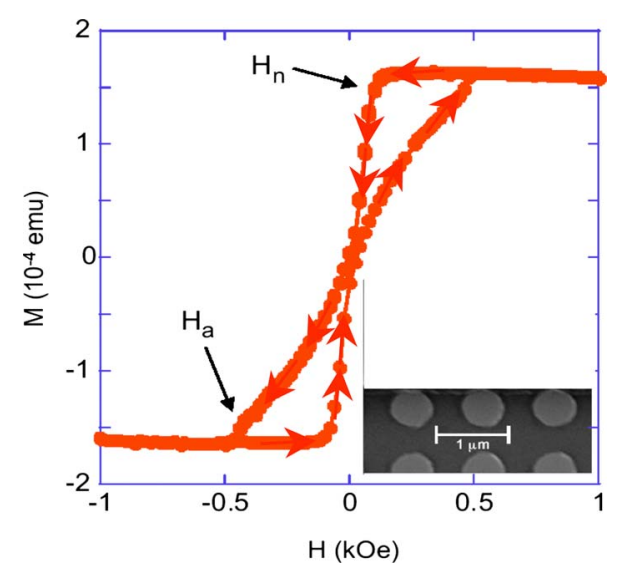

FIG. 1. (Color online) Magnetic hysteresis loop of the magnetic dot array as measured at $300 \mathrm{~K}$ with an in-plane magnetic field. The inset shows a scanning electron micrograph of a portion of the array. The arrows indicate the trajectories on sweeping from positive to negative fields and vice versa. (After Hoffmann et al.)

-80 and +450 Oe (and similarly between +80 and -450 Oe) the exact magnetic state depends on magnetic history; in particular, there is a kind of bistability where the magnetization can exit in either the high-field (essentially uniformly magnetized) phase or some presumed vortex-like phase(s), as will be discussed below.

Our ferromagnetic resonance data were taken with a broadband spectrometer and a sample cell designed to operate in a transmission mode. The cell itself consisted of a copper housing with input and output microwave coax connectors and an internal chamber that housed the Si chip (on which the disk array was patterned) clamped adjacent to a copper wire wound meander line which generates the $\mathrm{rf}$ magnetic fields; details concerning the construction of the cell have been presented elsewhere. ${ }^{15}$ The assembled cell was placed between the pole pieces of a 4" Varian electromagnet; the dc field was sensed with a Hall probe. The microwave signal was derived from a HP model 83623A synthesizer with a frequency range of $10-20 \mathrm{GHz}$ and a maximum power output of $25 \mathrm{dBm}$. This signal was fed to the cell through a copper coaxial line while a second line fed the cell output to a microwave diode detector. At this point one could, with a suitable offset, simply amplify and digitize the diode output vs magnetic field; this strategy was recently employed to perform a broadband FMR study on the same disk array in the frequency range $3-19 \mathrm{GHz}^{16}$ For the present work the standard field modulation technique was used, the ac field being generated by a pair of field coils surrounding the pole faces that were, in turn, driven by a power amplifier. A Princeton HR-8 lock-in detected the ac component of the diode output and in addition supplied the ac signal to the power amplifier. The output of the detector, which is nominally the field derivative of the FMR absorption line, and the output of the Hall probe were digitized and recorded by a computer. In addition to logging the raw data, the computer was programmed to perform the field integral of the lock-in output, thereby yielding the absorption line directly. For the low-field experiments performed here the magnet was driven by a 20 A Kepco operational amplifier

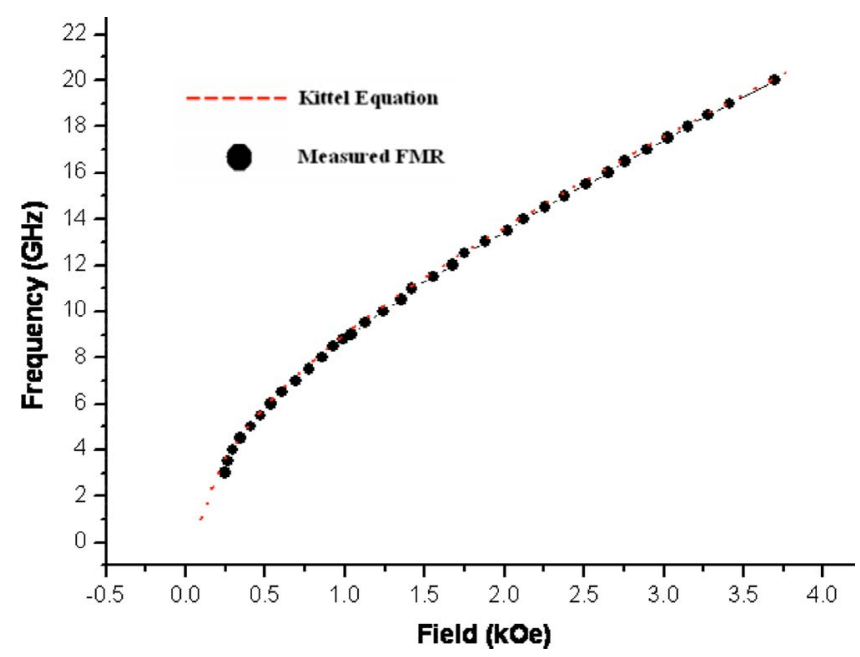

FIG. 2. (Color online) The measured field vs frequency behavior for the disk array for an in-plane magnetic field. The curve shows a fit to the Kittel equation using the parameters given in the text.

power supply, operating in a constant current mode that could sweep continuously at an adjustable rate between positive and negative currents.

FMR data were collected at $300 \mathrm{~K}$ on a Py disk array patterned in an essentially identical manner as the sample used by Hoffmann et al. The behavior over a broad range of frequencies and fields is shown in Fig. 2. For a uniformly magnetized film subjected to an in-plane magnetic field the data may be represented by the Kittel equation

$$
f=\frac{\gamma}{2 \pi} \sqrt{\left(H_{0}+H_{\mathrm{A}}\right)\left(H_{0}+H_{\mathrm{A}}+4 \pi M_{\mathrm{S}}\right)},
$$

where we have included the effects of a uniaxial anisotropy field, $H_{\mathrm{A}}$. We find that the data for our disk array can be accurately fitted to Eq. (1) with the following parameters: $\gamma / 2 \pi=2.92 \mathrm{GHz} / \mathrm{kOe}, 4 \pi M_{\mathrm{S}}=9.5 \mathrm{kOe}$, and $H_{\mathrm{A}}=-0.087 \mathrm{kOe}$, as shown in Fig. 2. The saturation magnetization is in the range typically observed for deposited films Py and the anisotropy field $H_{\mathrm{A}}$ can arise from substrate induced strain or may be associated with dipole interactions between disks.

Figure 3 shows the observed derivative of microwave absorption of the disk array at $4.2 \mathrm{GHz}$ as the field is swept between +900 and -900 Oe. Note that when sweeping from negative to positive fields a resonance is seen for negative fields whereas no resonance is seen for positive fields indicating the sample is in another state. The opposite behavior is obtained when sweeping from positive to negative fields.

We now examine the data at $4.2 \mathrm{GHz}$ in greater detail; essentially identical data were taken at $4.6 \mathrm{GHz}$ that will not be shown. Note the resonance at $4.2 \mathrm{GHz}$ frequency corresponds to a magnetic field that is less than $200 \mathrm{Oe}$; this field lies within the bistable interval observed in the magnetization experiments discussed above. The presence of a resonance (at a value in nominal agreement with the Kittel equation) implies an essentially uniformly magnetized film; we interpret the absence of a resonance to imply the disks are in a vortex-like phase, given that such phases are known to 


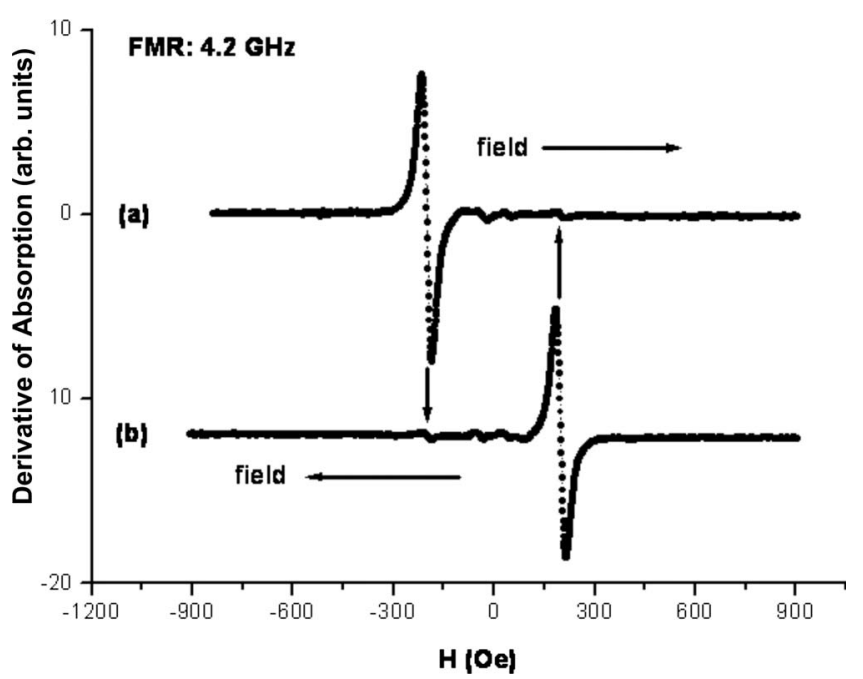

FIG. 3. The observed microwave absorption derivative for the disk array at $4.2 \mathrm{GHz}$ as the field is swept between +900 and -900 Gauss. Note that when sweeping from negative to positive fields a resonance is seen for negative fields whereas no resonance is seen for positive fields, indicating the sample is in another state. The opposite behavior is obtained when sweeping from positive to negative fields.

exist in disks at low fields. At this point it is apparent that by limiting the range over which we sweep the field starting from a saturated state we can use the amplitude of the FMR signal to trace out the disappearance and the appearance of the FMR line and so measure the boundary separating the vortex-like and the uniform phases. As noted above, and given that the fundamental resonance frequency of a vortex state differs greatly from that for a uniform state, we interpret the disappearance of the uniform mode as implying that the array enters some kind of vortex-like phase, the nature of which we expand on below.

Figure 4 shows the behavior associated with the disappearance of the FMR line. Starting with the sample saturated at a high negative value, the field is swept to a series of less negative and then positive fields, the values of which are given in the inset. On reaching each of these fields the sweep direction is reversed and the resonance recorded. The greater the excursion toward positive fields the greater the loss in signal amplitude on returning to the nominal resonant field near -155 Oe.

Figure 5 shows the behavior associated with the appearance of the FMR line. After again saturating the sample at a high positive value, the field is swept through zero field to various negative values (given in the inset) at which point it is then reversed back through zero and the increase in signal amplitude recorded. The greater the excursion into negative fields the greater the increase in signal amplitude on returning to the nominal resonant field near -155 Oe.

Figure 6(a) shows a sigmoidal fit to the peak intensity vs the excursion field when the FMR line is disappearing while Fig. 6(b) shows a similar fit derived from the data when the FMR line is appearing. We conclude that the bistable behavior lies between about 15 and 419 Oe. We take these boundaries to be in satisfactory agreement with the magnetization
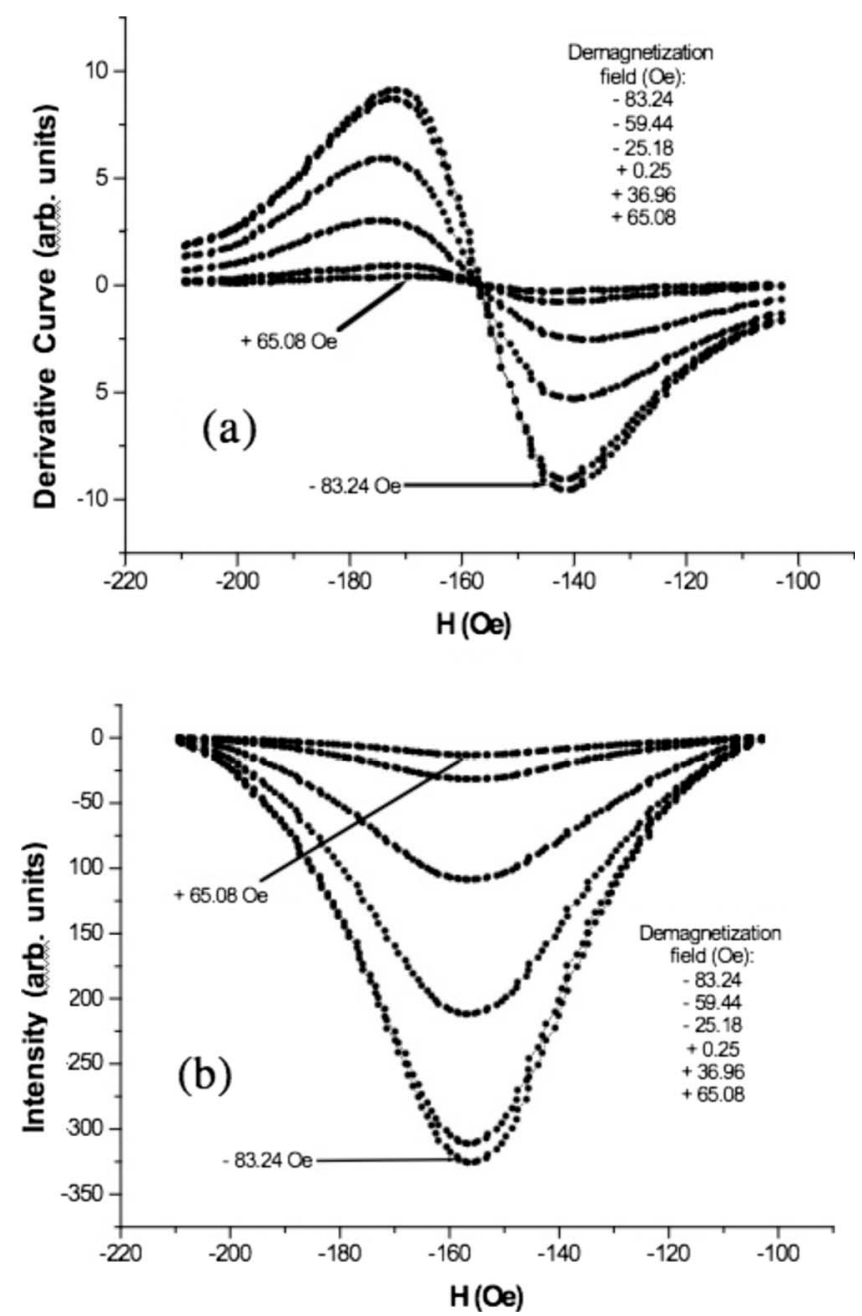

FIG. 4. The measured "disappearing behavior" of the FMR line for a frequency of $4.2 \mathrm{GHz}$. Starting with the sample saturated at a high negative field, the field is swept to a series of less negative and then positive fields (as given in the inset). The greater the excursion toward positive fields the greater the loss in signal amplitude on returning to the nominal resonant field near $-155 \mathrm{G}$.

data that were, after all, taken on a very similar, though different, sample.

We now discuss our micromagnetic simulations beginning with the field-dependent magnetization. At high fields a suitable initial configuration of the dipoles is to simply align them along the external field. One can then calculate the exchange and dipole field acting on each dipole arising from the remaining dipoles and reorient them to lie along the total local field. This procedure is continued until the dipole directions stabilize. The magnetization is obtained by summing the projection of each dipole on the external field direction. One can then lower the field and repeat the process, starting from the previous configuration and again iterating until the directions stabilize. Repeating this process we obtain the field dependence of the magnetization. We can check for metastability by choosing a random configuration of dipoles and dynamically relaxing them according to the LandauLifshitz equation in the presence of damping.

The resulting magnetization curve is shown in Fig. 7 to- 

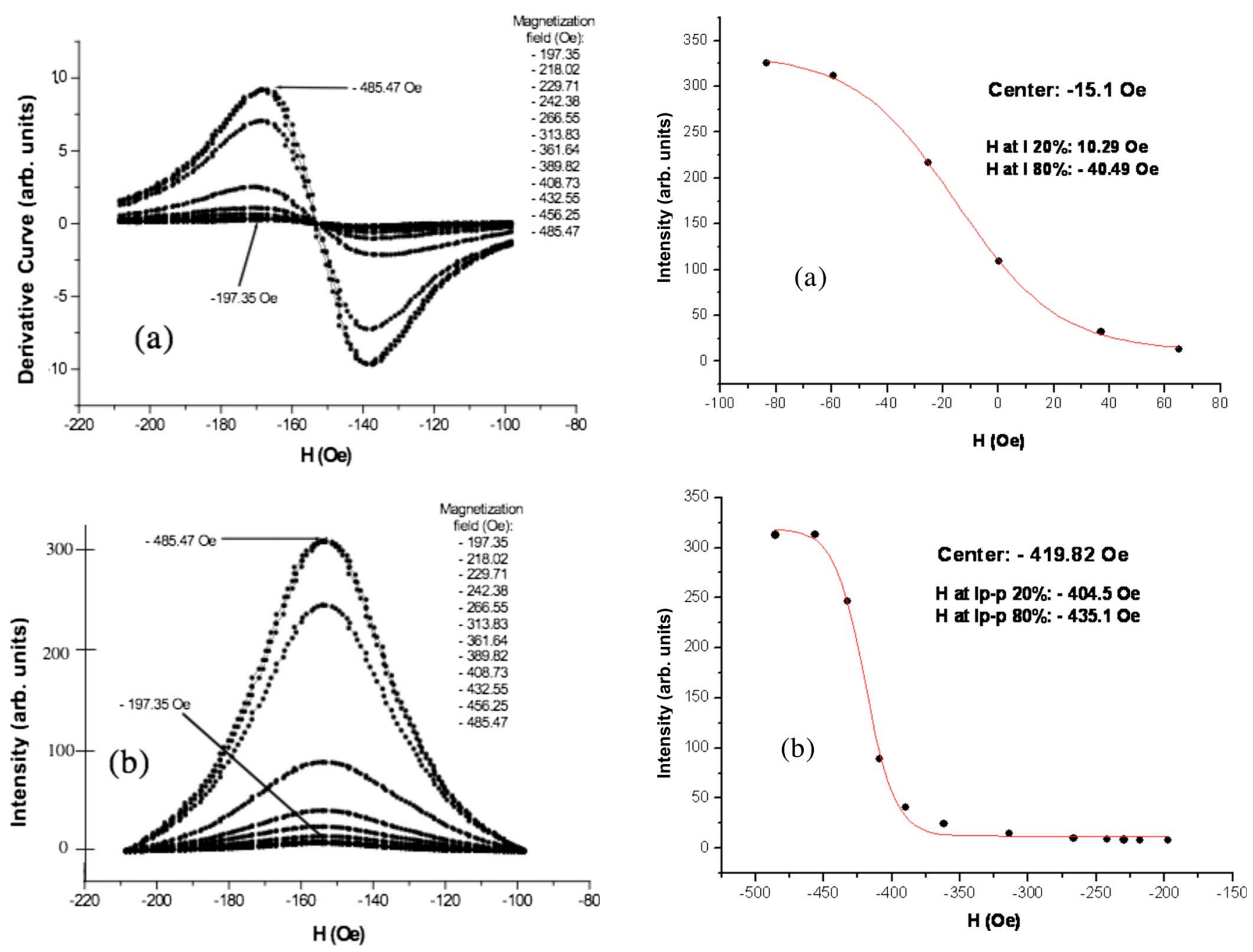

FIG. 5. The measured "appearing behavior" of the FMR line for a frequency of $4.2 \mathrm{GHz}$. After saturating the sample at a high positive value, the field is swept through zero to various negative values (given in the inset) at which point it is then reversed back through zero and the increase in signal amplitude recorded. The greater the excursion into negative fields the greater the increase in signal amplitude on returning to the nominal resonant field near $-155 \mathrm{G}$.

gether with the data of Hoffmann et al. ${ }^{17}$ On sweeping from positive to negative fields (right to left) the calculations predict a continuous transition at around 51 Oe from essentially uniformly magnetized disks to ones containing a double vortex; in the experiment a feature appears in the vicinity of +100 Oe which we may associate with this phenomenon. A sharp transition from a double-vortex to a single-vortex state occurs in the simulations near -15 Oe; we note that a distinct slope change occurs in the experiment near +15 Oe, which may be associated with this feature. Apart from this 30 Oe shift, the experiments show a much more gradual evolution between what the simulations identify as the uniform to two-vortex and the two-vortex to one-vortex transitions, which we comment on below. Finally, the simulations yield a sharp transition from a single vortex to the uniform state near -470 Oe while a more rounded feature is seen in the experiments starting around -400 Oe. Typical spin orientations in these uniform, double-vortex, and single-vortex states (the

FIG. 6. (Color online) (a) A sigmoid fit to the peak intensity of the appearing FMR line vs the excursion field. (b) The same for the disappearing FMR line.

latter at a nonzero field) are shown in Fig. 8.

We find that the numerically computed hysteresis curve depends sensitively on the "temperature" (which is numerically introduced as random flunctuations), and in addition changes somewhat from one run to another. This latter effect arises from a numerical artifact: the artificial pinning of a single vortex on the edge of the disks caused by the projection of a circular boundary onto the square discretization lattice used in the modeling. It turns out that there is a rather shallow energy barrier separating the two vortices cores in the double-vortex state; at higher temperatures the system easily overcomes this barrier, i.e., there is an activated transition to a single-vortex state.

Now in reality each of the dots has physical roughness, which would affect the vortex annihilation field and the observed behavior would then be an average over the patterned ensemble of disks, i.e., we expect the experimental data to contain an additional broadening arising from this effect.

These results of our FMR experiments can also be compared with the magnetization measurements of Hoffmann et $a l$. Given that the uniform to double-vortex transition is continuous (and hence reversible) we interpret our disappearing 


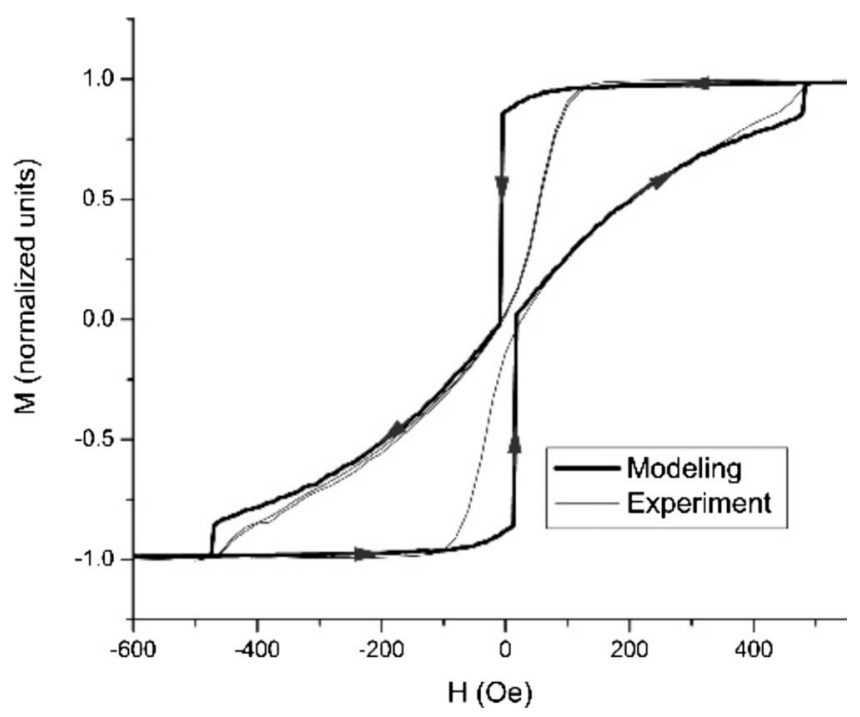

FIG. 7. The dashed lines show the measured field dependence of the magnetization while the heavy solid lines show the micromagnetic simulations. The arrows indicate the direction in which the field is swept.

field $H_{d}=15$ Oe as the irreversible double-to single-vortex transition which is to be compared with the large-to-small slope change in the magnetization that occurs at a similar field. We interpret $H_{a}$, which is also irreversible, as the single-vortex to uniform transition where the FMR and magnetization give -419 and -470 Oe, respectively. Note the larger disagreement among the experiments occurs for $H_{d}$; as noted above the variance in the simulations was largest here, which suggests that the experiments could be very sensitive to sample-to-sample variations. Some of these discrepancies with respect to the simulations may also arise from an uncertainty in $M_{\mathrm{S}}$; we used 700 Oe in the simulations whereas the value obtained above from fitting the Kittel equation was 756 Oe.

In summary we have used the appearance and disappearance of the ferromagnetic resonance line in a Py disk array to infer the boundaries of a single- and double-vortex state as it occurs in micromagnetic simulations. Both the data and the

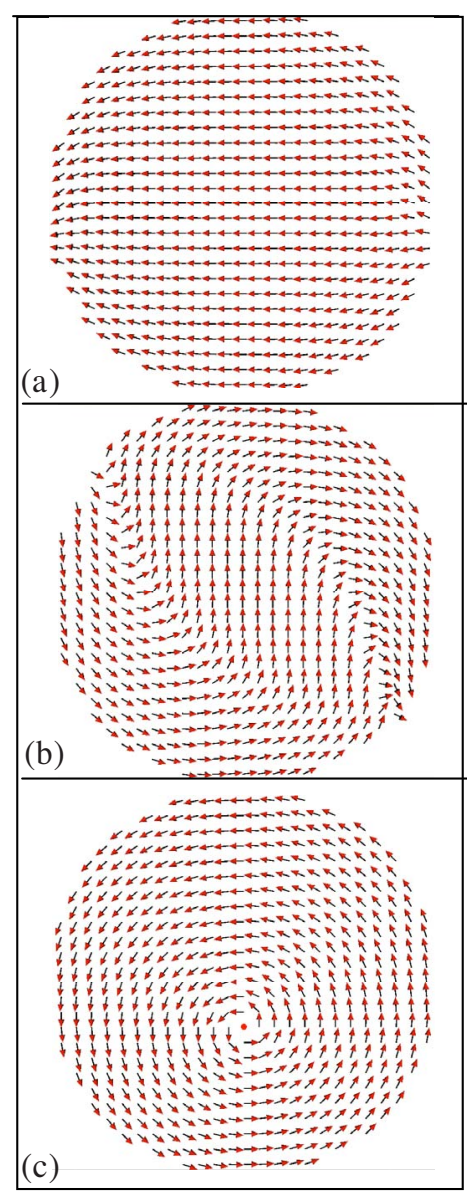

FIG. 8. (Color online) Typical arrangements of the spin vectors in a: (a) uniform, (b) double-vortex, and (c) single-vortex state, where the latter is in a nonzero external magnetic field that causes the vortex core to be displaced.

simulations are consistent with earlier magnetization studies on very similar arrays.

This work was supported by the National Science Foundation under Grants No. DMR-0509357, No. DMR0509691, No. ECCS-0823813, and No. ESI-0426328.
${ }^{1}$ S. Jung, B. Watkins, L. DeLong, J. B. Ketterson, and V. Chandrasekhar, Phys. Rev. B 66, 132401 (2002).

${ }^{2}$ K. Rivkin, W. T. Xu, L. E. De Long, V. V. Metlushko, and J. B. Ketterson, J. Magn. Magn. Mater. 309, 317 (2007).

${ }^{3}$ W. T. Xu, D. B. Watkins, L. E. DeLong, K. Rivkin, J. B. Ketterson, V. V. Metlushko, J. Appl. Phys. 95, 6645 (2004).

${ }^{4}$ C.-t. Yu, M. J. Pechan, and G. J. Mankey, Appl. Phys. Lett. 83, 3948 (2003).

${ }^{5}$ I. Guedes, N. J. Zaluzec, M. Grimsditch, C. Metlushko, P. Vavassori, B. Ilic, P. Neuzil, and R. Kumar, Phys. Rev. B 62, 11719 (2000).

${ }^{6}$ Object Oriented Micromagnetic Framework: math.nist.gov/ oommf/software.html
${ }^{7}$ www.rkmag.com/

${ }^{8}$ S. Jung, J. B. Ketterson, and V. Chandrasekhar, Phys. Rev. B 66 , 132405 (2002).

${ }^{9}$ K. Rivkin, A. Heifitz, P. R. Sievert, and J. B. Ketterson, Phys. Rev. B 70, 184410 (2004).

${ }^{10}$ K. Rivkin and J. B. Ketterson, J. Magn. Magn. Mater. 306, 204 (2006).

${ }^{11}$ K. Rivkin, W. Saslow, V. Chandrasehkhar, L. E. De Long, and J. B. Ketterson, Phys. Rev. B 75, 174408 (2007).

${ }^{12}$ K. Yu. Guslienko and A. N. Slavin, J. Appl. Phys. 87, 6337 (2000).

${ }^{13}$ V. Novosad, F. Y. Fradin, P. E. Roy, K. S. Buchanan, K. Y. Guslienko, and S. D. Bader, Phys. Rev. B 72, 024455 (2005). 
${ }^{14}$ A. Hoffmann, L. Fumagalli, N. Jahedi, J. C. Sautner, J. E. Pearson, G. Mihajlovic, and V. Metlushko, Phys. Rev. B 77, 060506(R) (2008).

${ }^{15}$ C. C. Tsai, J. Choi, Sunglae Cho, S. J. Lee, B. K. Sarma, C. Thompson, O. Chernyashevskyy, I. Nevirkovets, and J. B. Ketterson, Rev. Sci. Instrum. 80, 023904 (2009).
${ }^{16}$ I. P. Nevirkovets, O. Chernyashevskyy, J. B. Ketterson, V. Metlushko, and B. K. Sarma, J. Appl. Phys. 104, 063920 (2008).

${ }^{17}$ The data used here are the "raw" output from the original experiment. 